\title{
Exponential Increases in the Prevalence of Disability in the Oldest Old: A Canadian National Survey
}

\author{
Manon Guay ${ }^{a}$ Marie-France Dubois ${ }^{b}$ María Corrada ${ }^{d}$ \\ Marie-Pierre Lapointe-Garant ${ }^{c}$ Claudia Kawas $^{\mathrm{e}}$ \\ a School of Rehabilitation and ${ }^{b}$ Department of Community Health Sciences, Faculty of Medicine and Health Sciences, \\ Université de Sherbrooke, 'Étienne-Le Bel Clinical Research Center, Centre Hospitalier Universitaire de Sherbrooke, \\ Sherbrooke, Qué., Canada; ${ }^{d}$ Department of Neurology and Institute for Memory Impairments and Neurological \\ Disorders, e'Department of Neurobiology and Behavior, University of California, Irvine, Calif., USA
}

\section{Key Words}

Disability · Oldest old · Activities of daily living · Bathing

\begin{abstract}
Background: As most studies generally treat all $85+$ yearolds as a homogeneous group, little is known about the specific disabilities of the oldest old population, those aged 90 and older. Objective: To estimate age-specific prevalence of disability in activities of daily living for older Canadians, including the oldest old, those aged 90 and older. Methods: Cross-sectional national survey with a representative sample of noninstitutionalized Canadians aged between 50 and 104 years old $(n=28,406)$. Disability was self-reported and defined as needing assistance to perform self-care and domestic life activities. Results: The prevalence of disability increased with age, and the rise appeared exponential when considering the oldest old. At age 90 , the highest estimated rates of disability were reported for housekeeping (50\%), shopping (45\%) and transportation (44\%), and $21 \%$ reported requiring assistance for washing themselves. Compared to the 85-89 age group, the estimated proportion of people reporting disability in the $95+$ age group approximately triples for self-care activities and doubles for domestic life ac-
\end{abstract}

tivities. Conclusion: Even if we knew that disability increases with age, we can now state that it increases at an accelerated rate beyond age 85 . Grouping people aged $85+$ into one category leads to substantial underestimates of disability in the oldest old. Accurate estimates are necessary for adequate allocation of care and rehabilitation resources for a rapidly expanding age group.

(c) 2014 S. Karger AG, Basel

\section{Introduction}

If current life expectancy trends are maintained, over half of all babies born in developed countries today can be expected to live to be 100 years old [1]. Within the geriatric population, the number of people aged 90 and beyond - referred to as the oldest old - has grown both in quantity and in proportion $[2,3]$. Yet, this group is understudied [1-3], as are the disabilities they experience. In order to align services to the needs of this population, the prevalence and types of disabilities for specific activities and age groups must be clearly understood.

Typically, for the purpose of reporting on the prevalence of difficulty in everyday life, many different activi-

\section{KARGER}

E-Mail karger@karger.com

www.karger.com/ger
(C) 2014 S. Karger AG, Basel

0304-324X/14/0605-0395\$39.50/0
Dr. Manon Guay

School of Rehabilitation

Faculty of Medicine and Health Sciences, Université de Sherbrooke

Sherbrooke, QC J1H 5H4 (Canada)

E-Mail manon.guay@usherbrooke.ca 
ties are grouped [4]. Relying only on the usual distinction between basic activities of daily living (i.e. combining ADLs such as hygiene, dressing, eating, grooming or toileting) and instrumental ADLs (i.e. combining IADLs such as meal preparation, housekeeping or laundry) [5] for which components vary from one study to the other [6] does not provide sufficiently detailed information. The oldest old are at the limits of their functional capacity [7], and rehabilitation interventions intended to restore or maintain the independence of the elderly are very different from one activity to the next. Therefore, it is crucial to obtain a detailed picture of the prevalence of disability for specific activities of daily life.

When researchers do provide information on specific activities [8-10], 85+ year-olds are generally considered as a single group, which might fail to reveal or lead to misestimate the needs of the oldest old. To our knowledge, the only study that has reported on disability prevalence for oldest old Canadians is the Aging in Manitoba Longitudinal Study [11]. It was conducted more than 10 years ago and focused on Manitoba, which is only one of ten Canadian provinces. Thus, we need a current and comprehensive understanding of disability according to age. The purpose of our study is to estimate the age-specific prevalence of disability encountered in specific activities related to self-care and domestic life for Canadians aged 50 and beyond, including the oldest old population.

\section{Materials and Methods}

The Canadian Community Health Survey (CCHS) - Healthy Aging is a cross-sectional survey. The Canadian Health Statistics Division met with stakeholders to develop, refine and validate questionnaire content covering important health and social issues related to aging [12].

\section{Sampling}

The Healthy Aging Survey targeted Canadians living in private-occupied dwellings in any of the ten provinces. Canadians living in institutional dwellings, such as long-term care hospitals, nursing home, residences for senior citizens providing support services, were not included in the CCHS [13]. In Canada, the proportion of people living in institutional dwellings is $1.5 \%$ between 65 and 85 years old, and $10.1 \%$ over 85 years old [14]. Residents of Canadian territories, reserves and other Aboriginal settlements, remote regions, institutions, and full-time members of the Canadian Forces were excluded. With the 2006 Canadian Census as sampling frame, households were first randomly selected using a probability sampling strategy designed to guarantee representation of the ten provinces as well as urban/rural areas [14]. Using selection probabilities to ensure representation of five age groups (45-54, 55-64, 65-74, 75-84, 85+), one randomly selected respondent per household was then interviewed.

\section{Data Collection}

Trained interviewers visited the selected respondents at home to conduct face-to-face interviews between December 2008 and November 2009. Repeated visits to the dwelling were made at different times and on different days, either to set an appointment or to carry out the interview. Data collection by telephone was authorized only when translation was necessary. Another knowledgeable member of the household was allowed to answer questions for selected participants who were incapable of completing the interview due to physical or mental health reasons.

\section{Measures}

Function in self-care and domestic life activities was self-reported, using a three-level scale (Yes, without help/Yes, with help/ No) adapted from the OARS Multidimensional Assessment Questionnaire [15]. Activities for self-care (basics ADLs) were: washing oneself, grooming, dressing and eating. Activities of domestic life (IADLs) were: shopping, preparing meals, performing housework, using transportation, using a telephone, and taking medication. Activities relating to mobility (walking, transferring and toileting) were not included. For example, to assess ability in shopping, participants were asked the following question: 'Assuming you have transportation, can you go shopping for groceries or clothes without help (e.g. taking care of all shopping needs by yourself)? Can you go shopping for groceries or clothes with some help (e.g., you need someone to go with you on all shopping trips)? Are you completely unable to do any shopping?'

\section{Statistical Analyses}

Permission to release data collected through the Healthy Aging Survey is restricted as per the Statistics Act. To meet Statistics Canada requirements, variables most likely to lead to identification of an individual by the data analyst had to be deleted or collapsed to broader categories. Therefore, each function was dichotomized and a participant was defined as: (a) not disabled when no assistance was provided to perform a specific activity, and (b) disabled when the activity was performed with partial or total assistance.

The prevalence of disability was estimated for age groups 50 and older because prevalence was too low to be reported for people aged between 45 and 50. Since respondents aged 100 or beyond included only 16 women and no men, centenarians were included in the 95+ age category.

Survey weights were incorporated into the prevalence and variance estimates using the Bootvar program created by Statistic Canada [16]. Through a bootstrap method, this program produces estimates that are representative of the Canadian population and not just the sample itself. Sampling error associated with non-response was handled by adjusting the weight of persons who responded to the survey in order to compensate for those who did not respond. Older Canadians' characteristics are presented using weighted percentages. The prevalence of disability is presented according to 5-year age groups. Constructing 95\% confidence intervals (CI) made it possible to take into account sample variation.

To test whether disability increases with age in an exponential fashion, we also conducted nonlinear regression analyses. We estimated the equation $y=a+[b \cdot \exp (c \cdot$ age $)]$, where $y$ is the adjusted proportion of disabled Canadians and age is measured in years with the NLIN procedure. To examine the goodness of fit of our models, we calculated the Pseudo- $\mathrm{R}^{2}$ equal to $[1-$ (sum of squares of residual/sum of squares of corrected total)]. We fit two 
separate exponential models: one for self-care disability and another for domestic life disability. Disability in self-care was defined as requiring assistance with at least one self-care activity, and disability in domestic life as requiring assistance with at least three domestic life activities.

Analyses were performed with SAS software version 9.2 (SAS Institute, Cary, N.C., USA). The protocol was peer-reviewed by Statistics Canada to grant access to their data, but the opinions expressed in this paper do not represent the views of Statistics Canada.

\section{Results}

The response rate to the CCHS-Healthy Aging was $74.4 \%$, resulting in 28,406 participants aged 50 years or older. Canadians aged 50 and beyond were mostly women (52\%), Caucasian (86\%), living with someone (79\%), and residing in an urban area (84\%). Twenty-five percent did not have a high school diploma, and $36 \%$ had an annual family income of CAD $<50,000$.

Table 1 shows the estimated proportions (and 95\% CI) of home-dwelling Canadians with disability for each selfcare and domestic life activity studied. As expected, the prevalence of disability to perform specific day-to-day activities varies from one activity to another.

Among self-care activities, washing is the one with the highest prevalence of disability. Contrasting CI, one can see that the prevalence of disability for this activity is significantly different from that of the other self-care activities starting with the 70- to 74-year-old group. Regarding domestic life activities, about half of the people who had reached 90 were disabled in the following three activities: doing housework (50\%), shopping for groceries or clothes (45\%), and taking transportation (44\%), with $28 \%$ (95\% CI: $23-33 \%)$ being disabled in all three activities.

Results show an exponential rise in prevalence for age groups 85 and over. Figure 1 presents the fitted exponential curves relating age with disability prevalence in selfcare and domestic life activities. As shown by the Pseudo$\mathrm{R}^{2}$ statistic, the exponential model fits both the curves for prevalence of self-care activities $\left(\mathrm{R}^{2}=0.88\right)$ and prevalence of domestic life activities $\left(R^{2}=0.86\right)$. Figure 2 illustrates the pattern of disability prevalence in specific self-

\section{(Footnote to Table 1.)}

${ }^{a}$ Numbers are weighted to be representative of the Canadian population. ${ }^{b}$ Unweighted $n$ (weighted percentage). ${ }^{c}$ Prevalence of disability was too low to be reported. Figures in parentheses indicate $95 \% \mathrm{CI}$, unless otherwise stated.

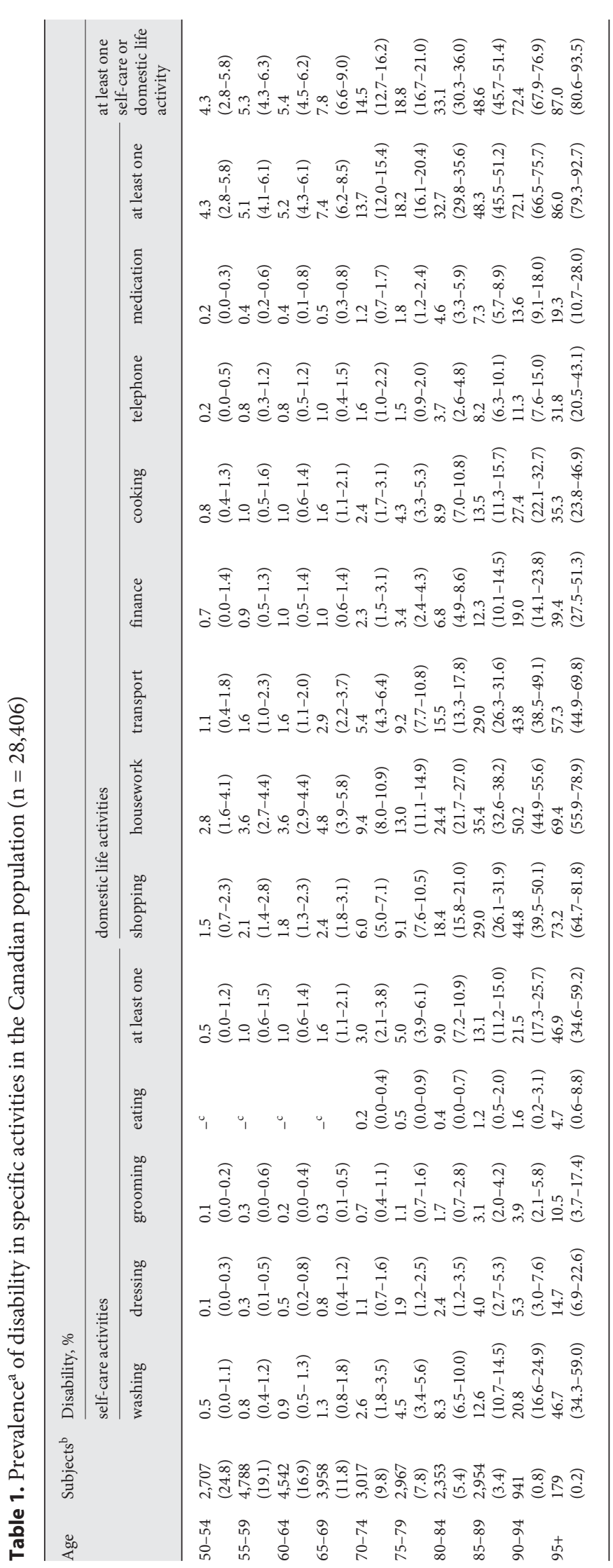

Gerontology 2014;60:395-401 DOI: $10.1159 / 000358059$ 


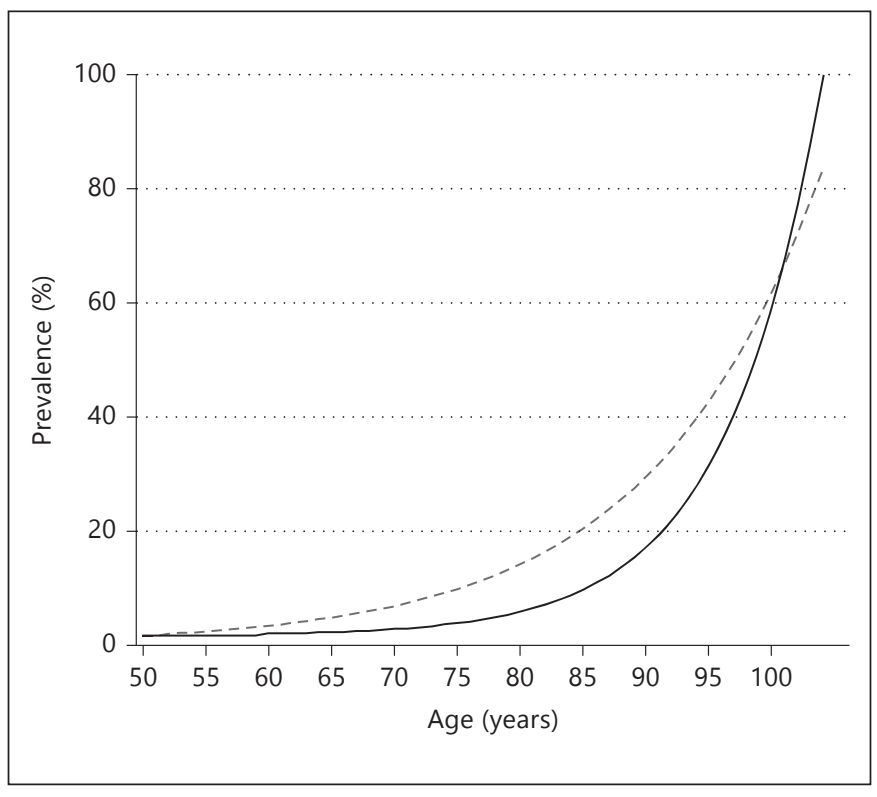

Fig. 1. Prevalence estimated with an exponential model of disability in day-to-day activities. Solid line: disability defined as requiring assistance with at least one self-care activity $\left(\mathrm{R}^{2}=0.88\right)$. Dashed line: disability defined as requiring assistance with at least three domestic life activities $\left(\mathrm{R}^{2}=0.86\right)$. Prevalence is weighted to be representative of the Canadian population.

care and domestic life activities across age groups. Compared to the 85-89 age group, the estimated proportion of people reporting disability in the 95+ age group approximately triples for self-care activities and doubles for domestic life activities.

Finally, a substantial proportion of people in the highest end of the age spectrum were not disabled in any activity. For example, we estimated that $79 \%$ of people aged between 90 and 94 who are living at home do not report needing assistance to wash, groom, and dress themselves or eat, and $28 \%$ report performing all of the eleven activities on their own.

\section{Discussion}

The purpose of this study was to estimate the age-specific prevalence of disability encountered in specific dayto-day activities for older Canadians, including the oldest old. For all activities studied, the prevalence of disability increased with age, and the increase was more notable after 85 years of age. Grouping people aged $85+$, or even $90+$, together leads to substantial underestimates of disabilities in the oldest old.
Our results obtained from a Canadian national survey highlight a profound increase in the prevalence of disability in the oldest old population. This finding is consistent with a US study of the oldest old, The 90+ Study, which found that $44 \%$ of 90 - to 94 -year-olds, $66 \%$ of 95 - to 99 -year-olds, and $92 \%$ of centenarians had difficulties with one or more ADLs [17]. The proportions of disability were higher in The 90+ Study than what the Canadian national survey shows, but those differences might be attributable to two methodological differences. First, the US study included transferring and walking indoors among ADLs, activities that are actually more related to the concept of mobility [18]. Since The 90+ Study reported that walking was the activity with the highest proportion of disability, including it among the ADLs examined leads to higher prevalence of disability in at least one ADL. Second, our study excluded people living in institutions, whereas The 90+ Study included these individuals and found that institutionalization was significantly associated with disability. In fact, when reexamining disability only among the noninstitutionalized participants of The $90+$ Study, the numbers indicate disability proportions of $24 \%$ for 90 - to 94 -year-olds and $55 \%$ for those aged 95 and over, which puts both results within our CI [unpubl. data].

Aging is strongly associated with the prevalence of disability in performing self-care and domestic life activities [19], and our results suggest that this association appears to be exponential rather than linear. Certainly, the prevalence of disability accelerates after 85 years of age. Even if the small size of samples is a common problem when studying the oldest age categories [3], it may still be preferable to either report disability separately in the very elderly, or refrain from formulating conclusions on disability in the oldest old, rather than grouping them with the younger elderly.

As found in several studies $[8,10,20,21]$, we confirmed that, among self-care activities, washing oneself is the activity for which the highest proportion of disability is reported across all age groups. Moreover, according to a longitudinal British study including oldest old persons, washing oneself is the activity with the earliest onset of disability; the median age of onset being 81.5 [22]. Clearly, with aging, washing oneself is a difficult self-care activity, and other interventions might be needed to prevent or reduce bathing disability besides the common recommendation to add grab bars and bath seat. It is uncertain that adding bath aids do forestall the subsequent development of disability [23], and it is unclear that current rehabilitation interventions meet the 
Fig. 2. Prevalence of disability in day-today activities. Self-care activities in black; domestic life activities in white. Prevalence is weighted to be representative of the Canadian population.

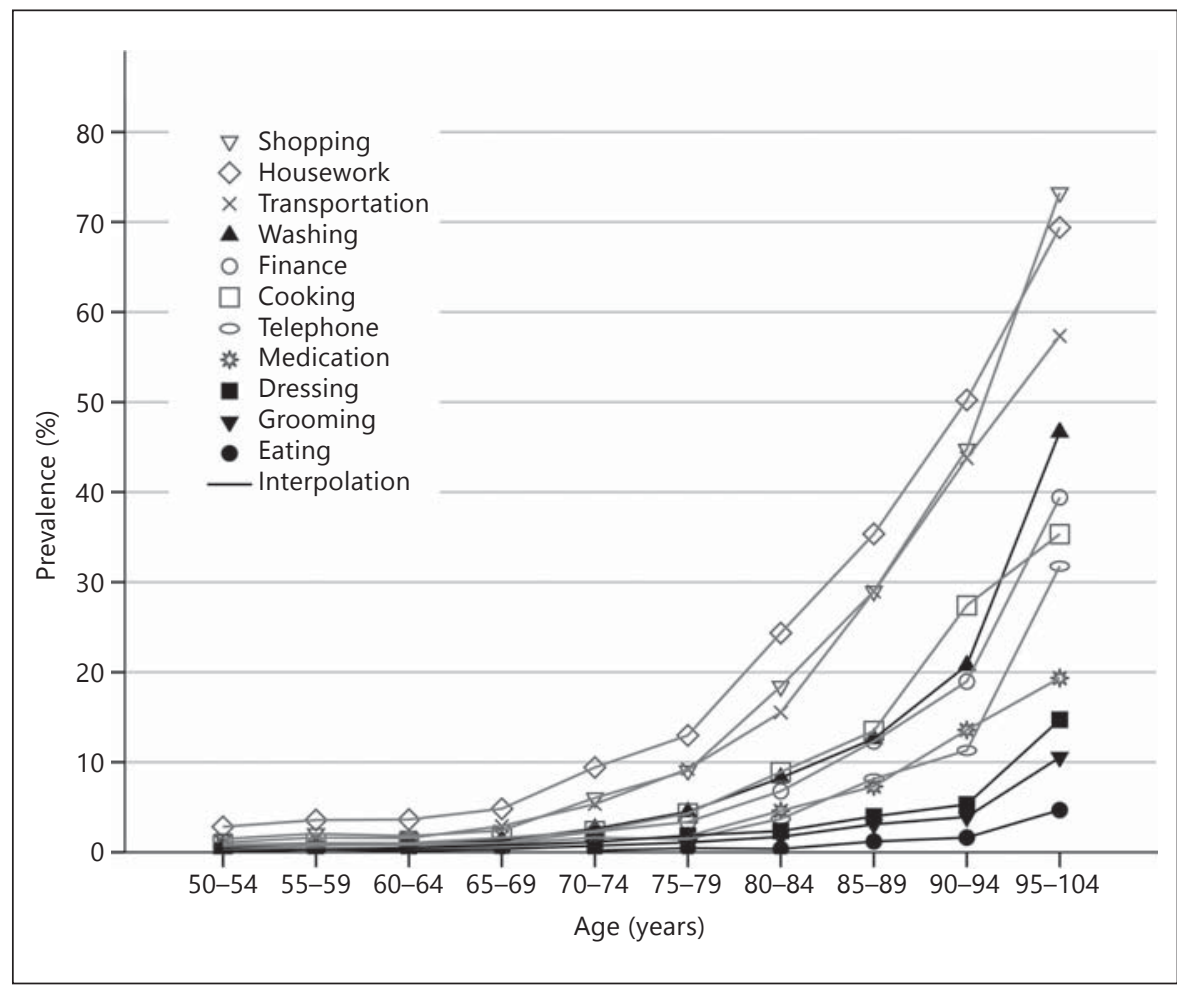

needs of the disabled elderly $[24,25]$. Because the elderly with unmet ADLs needs have higher rates of admission to acute care services [23], and washing oneself is the most challenging self-care activity, the focus on bathing should be increased when designing and studying rehabilitation services for the community-dwelling elderly.

The number of elderly who live to be 90 and beyond is growing. In 2012, $0.7 \%$ of the Canadian population was aged 90 or older, which represents 247,835 individuals [26]. The medium growth projection scenario indicates that the oldest old will represent $1.0 \%$ of the Canadian population in 2026 and $1.4 \%$, in 2036 [27]. As in other developed countries $[1,3]$, they are the fastest growing segment of the elderly population. Older persons are more likely to require paid personal assistance in their daily living [28], so the increasingly larger numbers of disabled oldest old will likely become an increasingly larger financial burden. However, similar to others [29], we did observe the existence of oldest old without disability. Studying the characteristics of those elderly could help us understand how to prevent or delay disability and contribute to the understanding of compression or expansion of morbidity concepts [30] associated with increases in life expectancy.

Prevalence of Disabilities for Older Canadians
Methodological constraints need to be acknowledged, as they might have led us to slightly misestimate disability in the Canadian population. For instance, because the capacity to perform each activity was self-reported, classification errors are possible. Moreover, $1.8 \%$ of the respondents reported having memory or problem-solving difficulties often or always, which sheds doubt on the accuracy of their answers and may induce a response bias. A few interviews (4.3\%) were done over the phone for translation purposes and could have resulted in misunderstandings. For $2.4 \%$ of the interviews, a knowledgeable member of the household answered the questionnaires in place of a participant unable to do so due to physical or mental incapacities. It has been shown that it may be reasonable to use a proxy to document disability in a survey on aging [31]; however, issues of possible bias remain [32]. Additionally, due to confidentiality concerns, the disability outcomes were dichotomized instead of analyzed according to the three levels documented in the survey (i.e. performing the activity without help vs. performing the activity with help vs. unable to perform the activity). More detailed information would have better captured disability as a continuum going from difficulty to dependence [33]. Our cross-sectional study design has limitations. Although it provides a portrait of 
elderly Canadians at a specific moment in time, it does not allow us to project future disability trends, which can be better defined by longitudinal data and can be affected by cohort effects and other trends. Finally, the estimated prevalence is based on a sample of Canadians living in the community. As the proportion of Canadians living in institutional dwellings is about $10 \%$ over the age of 85 years and institutionalization is associated with increased odds of disability [17], the estimated prevalence should not be generalized to all Canadians aged over 85 years old. However, because the proportion of Canadians living in institutional dwellings before the age of 85 years old is fairly low (1.5\%), our results provide a good portrait of the prevalence of disability for those age groups in Canada.

Nevertheless, this study has undeniable strengths. Reported prevalence of disability is based on a large sample representative of an entire country. Moreover, the random sampling strategy and the weighting of estimates provide prevalence estimates of disability in specific dayto-day activities that are more representative than estimates provided by cohort studies. Finally, we were able to study a wide range of ages and a variety of everyday activities, which brought to light essential information that can help enhance knowledge about a fundamental clinical aspect of aging and set targets for public health interventions.

\section{Conclusion}

Prevalence of disability varies between specific day-today activities for Canadians living at home. For all selfcare and domestic life activities studied, prevalence increases with age and increases faster after the age of 85 . Compared to the 85-89 age group, the estimated proportion of people who are disabled in the 95+ age group approximately triples reaching $47 \%$ for self-care activities (basic ADLs) and roughly doubles reaching about $86 \%$ for domestic life activities (IADLs). This article provides a portrait of disability in the Canadian older adult population and offers important information that can be taken into consideration by home-health practitioners and administrators when prioritizing services, including allocation of rehabilitation resources, to cope with the public health challenges of our aging population.

\section{Acknowledgements}

M.G. was awarded a postdoctoral fellowship by the Quebec Inter-University Center for Social Statistics to conduct this work in collaboration with The 90+ Study team at the Institute for Memory Impairment and Neurological Disorders, University of California, Irvine, USA. M.C. and C.K. are supported by the National Institute on Aging Grant R01AG21055.

\section{References}

1 Christensen K, Doblhammer G, Rau R, Vaupel J: Ageing populations: the challenges ahead. Lancet 2009;374:1196-1208.

2 Kawas C: The oldest old and the 90+ Study. Alzheimers Dement 2008;4:S56-S59.

3 He W, Muenchrath M: 90+ in the United States: 2006-2008. Washington, US Census Bureau, 2011. www.census.gov/prod/2011 pubs/acs-17. pdf (accessed May 19, 2013).

4 Winblad I, Jaaskelainen M, Kivela S, Hiltunen P, Laippala P: Prevalence of disability in three birth cohorts at old age over time spans of 10 and 20 years. J Clin Epidemiol 2001;54:1019-1024.

5 Katz S: Assessing self-maintenance: activities of daily living, mobility, and instrumental activities of daily living. J Am Geriatr Soc 1983; 31:721-727.

6 6 Buurman B, van Munster B, Korevaar J, de Haan R, de Rooij S: Variability in measuring (instrumental) activities of daily living functioning and functional decline in hospitalized older medical patients: a systematic review. J Clin Epidemiol 2011;64:619-627.

7 Baltes P, Smith J: New frontiers in the future of aging: from successful aging of the young old to the dilemmas of the fourth age. Gerontology 2003;49:123-135.
8 Chan K, Kasper J, Brandt J, Pezzin L: Measurement equivalence in ADL and IADL difficulty across international surveys of aging: findings from the HRS, SHARE, and ELSA. J Gerontol B Psychol Sci Soc Sci 2012;67:121132.

9 LaPlante M: The classic measure of disability in activities of daily living is biased by age but an expanded IADL/ADL measure is not. J Gerontol B Psychol Sci Soc Sci 2010;65:720732.

10 Naik A, Concato J, Gill T: Bathing disability in community-living older persons: common, consequential, and complex. J Am Geriatr Soc 2004;52:1805-1810.

11 Finlayson M, Havens B: Changes over time in long-term care use, ADL and IADL among the oldest-old participants of the aging in Manitoba longitudinal study. Can J Aging 2001;20:271-290.

12 Canadian Community Health Survey Healthy Aging (CCHS). Statistics Canada. http://www23.statcan.gc.ca/imdb/p2SV.pl? Function $=$ getSurvey $\&$ SDDS $=5146 \&$ Item $\mathrm{Id}=47962$ \&lang=en (accessed May 19, 2013).

13 Collective dwelling. 2011. http://www12.stat can.gc.ca/census-recensement/2011/ref/dict/ dwelling-logements002-eng.cfm (accessed September 24, 2013).

14 Age Groups, Sex and Living Arrangements for the Population in Collective Dwelling, Residence for senior Citizens of Canada. 2011. http://www12.statcan.gc.ca/censusrecensement/2011/dp-pd/tbt-tt/Rp-eng.cfm? tabid $=1$ \&lang $=e \& a=r \&$ apath $=3 \&$ detail $=0 \&$ di $\mathrm{m}=0 \& \mathrm{fl}=\mathrm{a} \&$ free $=0 \& \mathrm{gc}=0$ \&gid $=906040 \& \mathrm{gk}=$ $0 \& \operatorname{grp}=1 \& \mathrm{o}=\mathrm{d} \&$ pid $=104097 \&$ prid $=0$ \&ptype $=101955 \& \mathrm{~s}=0 \&$ showall $=0 \& \mathrm{sub}=0 \&$ temporal $=2011 \&$ theme $=91 \&$ vid $=0$ \&vnamee $=\&$ vname $\mathrm{f}=\& \mathrm{~d} 1=0 \& \mathrm{~d} 2=0 \& \mathrm{~d} 3=0 \& \mathrm{~d} 4=0 \& \mathrm{~d} 5=0 \& \mathrm{~d} 6=0$ (accessed September 24, 2013).

15 Fillenbaum G, Smyer M: The development, validity, and reliability of the OARS multidimensional functional assessment questionnaire. J Gerontol 1981;36:428-434.

16 Bootvar User Guide (Bootvar 3.2-version SAS). Statistics Canada. http://www.statcan. gc.ca/rdc-cdr/bootvar_sas-eng.htm (accessed May 19, 2013).

17 Berlau D, Corrada M, Kawas C: The prevalence of disability in the oldest-old is high and continues to increase with age: findings from The 90+ Study. Int J Geriatr Psychiatry 2009; 24:1217-1225. 
18 International Classification of Functioning, Disability and Health (ICF). World Health Organization. http://apps.who.int/classifications/ icfbrowser/ (accessed May 19, 2013).

19 Stuck A, Walthert J, Nikolaus T, Büla C, Hohmann C, Beck J: Risk factors for functional status decline in community-living elderly people: a systematic literature review. Soc Sci Med 1999;48:445-469.

20 Gill T, Guo Z, Allore H: The epidemiology of bathing disability in older persons. J Am Geriatr Soc 2006;54:1524-1530.

21 Berlau D, Corrada M, Peltz C, Kawas C: Bathing as a potential target for disability reduction in the oldest old. A J Public Health 2011; 101:200-201.

22 Jagger C, Arthur A, Spiers N, Clarke M: Patterns of onset of disability in activities of daily living with age. J Am Geriatr Soc 2001;49: 404-409.

23 Gill T, Han L, Allore H: Bath aids and the subsequent development of bathing disability in community-living older persons. J Am Geriatr Soc 2007;55:1757-1763.
24 Murphy S, Gretebeck K, Alexander N: The bath environment, the bathing task, and the older adult: a review and future directions for bathing disability research. Disabil Rehabil 2007;29:1067-1075.

25 Zingmark M, Bernspang B: Meeting the needs of elderly with bathing disability. Aust Occup Ther J 2011;58:164-171.

26 Population by sex and age group. Statistics Canada. http://www.statcan.gc.ca/tablestableaux/sum-som/101/cst01/demo10a-eng. htm (accessed May 19, 2013).

27 Projected population by age group and sex according to three projection scenarios for 2010, 2011, 2016, 2021, 2026, 2031 and 2036, at July 1. 2013. Statistics Canada. http://www. statcan.gc.ca/tables-tableaux/sum-som/101/ cst01/demo23g-eng.htm\# (accessed May 19, 2013).
28 LaPlante M, Harrington C, Kang T: Estimating paid and unpaid hours of personal assistance services in activities of daily living provided to adults living at home. Health Serv Res 2002;37:397-415.

29 Donald I, Foy C, Jagger C: Trends in disability prevalence over 10 years in older people living in Gloucestershire. Age Ageing 2010; 39:337-342.

30 Jagger C: Compression or expansion of morbidity - what does the future hold? Age Ageing 2000;29:93-94.

31 Ostbye T, Tyas S, McDowell I, Koval J: Reported activities of daily living: agreement between elderly subjects with and without dementia and their caregivers. Age Ageing 1997; 26:99-106.

32 Santos-Eggimann B, Zobel F, Berod A: Functional status of elderly home care users: do subjects, informal and professional caregivers agree? J Clin Epidemiol 1999;52:181-186.

33 Gill T: Assessment of function and disability in longitudinal studies. J Am Geriatr Soc 2010;58(suppl 2):S308-S312. 